\title{
The brains of the bones: how osteocytes use WNT1 to control bone formation
}

\author{
Frank Rauch \\ Shriners Hospital for Children and McGill University, Montreal, Quebec, Canada.
}

\begin{abstract}
WNT proteins drive the development and maintenance of many tissues, including bone. It is less clear which of the many WNT proteins act on bone or where these WNTs act in the skeleton; however, loss-of-function mutations in WNT1 cause bone fragility in children and adults. In this issue of the $J C I$, Joeng and colleagues demonstrate that bone formation is under the control of WNT1 produced by osteocytes, the cells that reside deep in the bone matrix and form dendritic networks. The implication of WNT1 in the control of bone formation identifies a potential new target for the treatment of low bone mass disorders, such as osteoporosis.
\end{abstract}

\section{Which WNT, where?}

A role for WNT signaling in bone development and maintenance was first discovered through the study of osteoporosis pseudoglioma syndrome, an ultra-rare disease that is characterized by blindness, low bone mass, and frequent fractures in children (1). This disorder is caused by homozygous loss-of-function mutations in the gene encoding LDL receptor-related protein 5 (LRP5), which acts as a coreceptor for WNT ligands on the surface of osteoblasts, the cells responsible for bone formation. When WNT ligands bind to LRP5, bone formation is stimulated; therefore, mutations that inactivate LRP5 lead to slow bone formation and low bone mass.

This basic machinery was discovered more than 15 years ago, but it is still unclear which of the many WNT proteins play an important role in human bone. Studies in children with another rare bone fragility disorder provided an answer. Specifically, homozygous inactivating mutations in WNT1 lead to a severe form of recessive osteogenesis imperfecta that is characterized by frequent fractures in early childhood and, in some patients, structural brain abnormalities (2-5). Approximately 20 individuals with homozygous
WNT1 mutations have been reported to date. Bone and brain phenotypes similar to those seen in these children are also present in the swaying mouse, which has a naturally occurring Wnt1 mutation (6). Heterozygous WNT1 mutations give rise to less severe bone fragility and manifest as early-onset osteoporosis in adolescents or young adults $(2,4)$.

Mouse studies have indicated that the WNT1-expressing bone cells are osteocytes, rather than bone-forming osteoblasts or bone-resorbing osteoclasts (2). Osteocytes are former osteoblasts that have become embedded in mineralized bone matrix (7), where they maintain contact with each other, and with cells on the bone surface, via canalicular canals through which they project their dendrites. This canalicular system also allows for a remarkably close contact between osteocytes and bone matrix: the combined length of canaliculi in a cubic centimeter of mineralized bone amounts to $74 \mathrm{~km}$, with $80 \%$ of the bone matrix located within $3 \mu \mathrm{m}$ of the nearest canaliculus (8). It is thought that osteocytes monitor the deformation of bone matrix that results from the exposure of the skeleton to mechanical forces and thus play an essential role

Related Article: p. 2678

Conflict of interest: The author has declared that no conflict of interest exists.

Reference information: J Clin Invest. 2017;127(7):2539-2540. https://doi.org/10.1172/JCI95386. 
ated the high-bone-mass phenotype. Conversely, genetic stimulation of mTORC1 signaling in osteocytes of the swaying mouse increased bone mass and prevented spontaneous fractures. Thus, mTORC1 signaling at least in part mediates the effect of WNT1 signaling on osteoblasts.

Finally, Joeng et al. showed that anti-sclerostin antibody treatment of the swaying mouse increased bone mass, improved mechanical bone properties, and decreased the fracture rate (9). These observed improvements in the swaying mouse following anti-sclerostin administration are significant on both mechanistic and translational levels. Sclerostin is an osteocyte-secreted protein that decreases WNT signaling and thereby inhibits bone formation. It does so by interacting with LRP5 on the surface of osteoblasts, which prevents the binding of WNT ligands to LRP5. Anti-sclerostin antibody treatment therefore has bone anabolic properties. The fact that the anabolic effect of anti-sclerostin therapy is still seen in the absence of WNT1 indicates that other WNT ligands are available in the bone environment to signal through LRP5. Potential candidates for such a compensatory mechanism include WNT7b and WNT10b, which both enhance bone formation and interact with LRP5 (10, 11). Nevertheless, WNT1 seems to be the predominant WNT ligand in this context, as the results by Joeng et al. show that anti-sclerostin treatment is unable to completely restitute bone mass in WNT1-deficient mice (9).

From a translational perspective, the preclinical studies by Joeng et al. suggest that treatment with anti-sclerostin antibody is worth exploring as a potential therapeutic strategy for bone fragility caused by WNT1 mutations. At present, antiresorptive therapy with bisphosphonates is the most widely used approach for treating bone fragility disorders. Unfortunately, this approach is not very effective in patients with WNT1 mutations $(4,12)$. Anti-sclerostin antibody treatment may thus become a more effective treatment option in the presence of WNT1 mutations. Another bone anabolic approach is treatment with teriparatide (parathyroid hormone 1-34), which decreases sclerostin expression in osteocytes (7). Interestingly, recent pilot studies showed encouraging results with teriparatide treatment of adults who had osteoporosis caused by heterozygous WNT1 mutations (13).

\section{Future directions}

In their series of elegant experiments, Joeng et al. have established that WNT1 production in osteocytes plays a key role in the control of bone formation and bone mass. This leads to the question of what drives WNT1 production in osteocytes under physiological conditions. Is WNT1 part of the mechanostat machinery, the putative system whereby osteocytes adapt bone strength to local mechanical requirements, or is osteocytic WNT1 under the control of systemic hormones?

The results by Joeng et al. also make WNT1 a potential player in bone disorders characterized by dysfunctional osteocytes and abnormal bone mass. For example, in X-linked hypophosphatemic rickets, which is caused by phosphate-regulating endopeptidase homolog, X-linked (PHEX) mutations, the bone matrix surrounding osteocytes is hypomineralized, but overall bone mass is paradoxically increased (14). In osteogenesis imperfecta, which is caused by mutations in collagen type Iencoding genes, osteocyte density is conspicuously increased, and bone mass is typically very low (15). Disordered WNT1 signaling might contribute to the bone mass abnormalities in these conditions.

Finally, the results by Joeng and colleagues offer new perspectives for the treatment of common bone mass disorders, such as osteoporosis. Pharmacological interventions to target WNT1 production specifically in osteocytes or to modify WNT1dependent signaling pathways in osteoblasts could become promising therapeutic avenues. Studies of extremely rare disorders thus may help treat common ones.

\section{Acknowledgments}

The author would like to thank Pierre Moffatt (Shriners Hospital for Children, Mon- treal, Quebec, Canada) for helpful feedback on this Commentary.

Address correspondence to: Frank Rauch, Shriners Hospital for Children, 1003 Decarie, Montreal, Quebec, Canada H4A OA9. Phone: 514.842.5964; Email: frauch@ shriners.mcgill.ca.

1. Gong Y, et al. LDL receptor-related protein 5 (LRP5) affects bone accrual and eye development. Cell. 2001;107(4):513-523.

2. Laine CM, et al. WNT1 mutations in early-onset osteoporosis and osteogenesis imperfecta. N Engl J Med. 2013;368(19):1809-1816.

3. Fahiminiya S, Majewski J, Mort J, Moffatt P, Glorieux FH, Rauch F. Mutations in WNT1 are a cause of osteogenesis imperfecta. JMed Genet. 2013;50(5):345-348.

4. Keupp K, et al. Mutations in WNT1 cause different forms of bone fragility. Am J Hum Genet. 2013;92(4):565-574.

5. Pyott SM, et al. WNT1 mutations in families affected by moderately severe and progressive recessive osteogenesis imperfecta. Am J Hum Genet. 2013;92(4):590-597.

6. Joeng KS, et al. The swaying mouse as a model of osteogenesis imperfecta caused by WNT1 mutations. Hum Mol Genet. 2014;23(15):4035-4042.

7. Plotkin LI, Bellido T. Osteocytic signalling pathways as therapeutic targets for bone fragility. Nat Rev Endocrinol. 2016;12(10):593-605.

8. Repp F, et al. Spatial heterogeneity in the canalicular density of the osteocyte network in human osteons. Bone Rep. 2017;6:101-108.

9. Joeng KS, et al. Osteocyte-specific WNT1 regulates osteoblast function during bone homeostasis. JClin Invest. 2017;127(7):2678-2688.

10. Chen J, et al. WNT7B promotes bone formation in part through mTORC1. PLoS Genet. 2014;10(1):e1004145.

11. Esen E, Chen J, Karner CM, Okunade AL, Patterson BW, Long F. WNT-LRP5 signaling induces Warburg effect through mTORC2 activation during osteoblast differentiation. Cell Metab. 2013;17(5):745-755.

12. Palomo T, et al. Skeletal characteristics associated with homozygous and heterozygous WNT1 mutations. Bone. 2014;67:63-70.

13. Välimäki VV, et al. Teriparatide treatment in patients with WNT1 or PLS3 mutation-related early-onset osteoporosis: A pilot study. JClin Endocrinol Metab. 2017;102(2):535-544.

14. Cheung M, et al. Cortical and trabecular bone density in X-linked hypophosphatemic rickets. JClin Endocrinol Metab. 2013;98(5):E954-E961.

15. Jones SJ, Glorieux FH, Travers R, Boyde A. The microscopic structure of bone in normal children and patients with osteogenesis imperfecta: a survey using backscattered electron imaging. Calcif Tissue Int. 1999;64(1):8-17. 